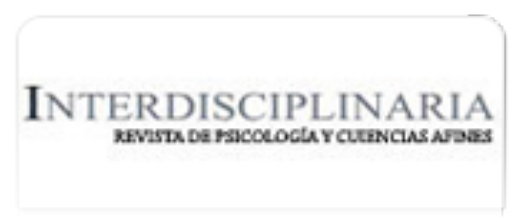

Interdisciplinaria

ISSN: 0325-8203

ISSN: 1668-7027

interdisciplinaria@fibercorp.com.ar

Centro Interamericano de Investigaciones Psicológicas y

Ciencias Afines

Argentina

Mustaca, Alba Elisabeth; Franco, Paul

Percepción de estudiantes y graduados sobre el estatus de la Psicología en Argentina

Interdisciplinaria, vol. 35, núm. 2, 2018, Julio-, pp. 327-340

Centro Interamericano de Investigaciones Psicológicas y Ciencias Afines

Argentina

Disponible en: https://www.redalyc.org/articulo.oa?id=18058785006

Cómo citar el artículo

Número completo

Más información del artículo

Página de la revista en redalyc.org

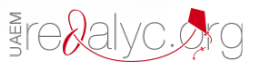

Sistema de Información Científica Redalyc Red de Revistas Científicas de América Latina y el Caribe, España y Portugal Proyecto académico sin fines de lucro, desarrollado bajo la iniciativa de acceso abierto 


\title{
Percepción de estudiantes y graduados sobre el estatus de la Psicología en Argentina*
}

\section{Perception of students and graduates on status of Psychology in Argentina}

\author{
Alba Elisabeth Mustaca** y Paul Franco***
}

\begin{abstract}
*La presente investigación fue parcialmente financiada por la Facultad de Psicología y Humanidades de la Universidad Abierta Interamericana (UAI).

**Doctora en Psicología. Fundadora del Laboratorio de Psicología Experimental y Aplicada (PSEA-IDIMCONICET). Directora de proyectos de investigación del Consejo Nacional de Investigaciones Científicas y

Tecnológicas (CONICET), Secretaría de Ciencia y Tecnología (SECYT) y de la Universidad Abierta Interamericana (UAI). Investigadora del Centro de Altos Estudios en Ciencias Humanas y de la Salud (CAESIHS- UAI). Profesora e investigadora de la Facultad de Psicología (UAI) y docente de cursos de especialización. E-mail: albamustaca@gmail.com; alba.mustaca@uai.edu.ar

***Licenciado en Psicología, docente de la Universidad Abierta Interamericana (UAI) e investigador del Centro de Altos Estudios en Ciencias Humanas y de la Salud (CAECIHS). Participa en varios proyectos de investigación relacionados con la psicología ambiental y la enseñanza de la Psicología.

E-mail: rimbaud64@gmail.com
\end{abstract}

Los autores agradecen al alumno Nicolás Alonso por su participación en la administración del cuestionario a la mayoría de los estudiantes.

Facultad de Psicología y Humanidades. Universidad Abierta Interamericana (UAI).

Ciudad Autónoma de Buenos Aires, Argentina.

\section{Resumen}

Hay evidencia que muestra que existen diferencias entre estudiantes y graduados sobre los puntos de vista acerca de la naturaleza de la Psicología. El objetivo del trabajo es evaluar la percepción sobre el status de la disciplina entre estudiantes y graduados de Psicología de Argentina con la Escala de Psicología como ciencia, basada en la de Friedrich (1996). Consiste en 20 afirmaciones que se deben puntuar con una escala Likert, que va desde completamente en desacuerdo (1) a completamente de acuerdo (7), clasificadas en tres factores: F1, la Psicología es una disciplina análoga a la Biología o la Física; F2, la investigación llevada a cabo en entornos controlados de laboratorio es esencial para la comprensión de la conducta cotidiana, y F3, la conducta es predecible. El Factor 4 contiene ítems de relleno para ensombrecer el objetivo del cuestionario. La Escala se envió mediante un sistema on line y en forma presencial. Los graduados presentaron puntajes significativamente mayores que los estudiantes en los tres factores de interés. Los graduados de orientación cognitivaconductual obtuvieron un puntaje medio que indica que están muy de acuerdo o de acuerdo con el estatus científico de la Psicología a diferencia de los estudiantes y psicoanalistas. Además, los estudiantes de los primeros años presentan puntajes significativamente más altos en F1 y F3 en relación a los de los últimos años. Los resultados se discuten en relación a la enseñanza de la Psicología.

Palabras clave: Enseñanza de la Psicología; Ciencia; Estudiantes; Graduados 


\begin{abstract}
One of the fundamental goals of education is for people to learn to differentiate scientific knowledge from the one derived from common sense and pseudoscience. Learning to think scientifically is considered an easy task. It is not natural to humans; it requires changing certain structures of knowledge towards notions that need reflection, and an important transformation of some conceptual networks that are involved in the information processing mechanisms natural of all living organisms. In the case of psychology, the conceptual change from folk psychology (or common sense) and pseudoscience to psychological science is also difficult to teach, perhaps even more so than in physics or biology, as the latter are more developed, more remote from human problems and there are less epistemological controversies about their methodology. There is evidence of differences between students and graduates on their views about the scientific basis of psychology. In this paper, the perception of psychology's status was evaluated among psychology students and graduates from universities in Argentina using the Psychology as a Science Scale (Friedrich, 1996 PAS). It's comprised of 20 items with a 7 point Likert style response ranging from 1 (strongly disagree) to 7 (strongly agree). It is divided into three factors. F1: psychology can be considered as a science similar to biology or physics (4 items, e.g. A degree in psychology should be a Bachelor of Science rather than one in Arts or Humanities); F2: research conducted in controlled laboratory environments is essential for the understanding of everyday behavior (5 items, e.g. Being able to do experiments is as important to psychology students as for chemistry, physics or biology students); and F3: behavior is predictable (6 items, e.g. Although each person is unique, it is possible for science to find general laws to explain human behavior). It also has 4 filler questions (FR) to hide the objectives of the survey and control social desirability (eg. An introductory course in psychology should encompass the widest range of possible topics). Friedrich (1996) reported that the scale has an internal consistency coefficient alpha of .71 for a sample of students and Holmes (2014), repor-
\end{abstract}

ted alpha of .74 for graduate / teachers. For the sample presented, Cronbach's alpha was .74 for all items. The Spanish translation was done by the authors of the study and evaluated by two experts in psychology and English proficiency. Then it was administered to a group of psychology students to confirm the understanding of the items. The survey was administered both through an online system and in person. The main results show that students have significantly lower score than graduates in the three factors of interest. However, graduates who identify with a cognitive-behavioral framework or with an integrative-eclectic approach strongly agree or agree with the scientific status of psychology as measured in F1, F2 and F3, unlike students and psychoanalysts who scored significantly less in F1 and F3. Moreover, students of the first years of study have a significant minor score than students of the last years on F1 and F3 $(p<.05)$. This work has as limit that the sample is not representative and the questionnaire was not validated in its version in Spanish. However, the results are similar to those of other countries, although it was the first that discriminated the opinion of the graduates in function of the theoretical orientation. Future research confirms these results. These results are discussed in relation to studies in other countries and to the teaching of psychology.

Key words: Teaching of Psychology; Science; Students; Graduates.

\section{Introducción}

Uno de los objetivos fundamentales de la educación es que las personas aprendan a diferenciar el conocimiento científico del derivado del sentido común y de las pseudociencias. El primero, producto de la cotidianidad, se refiere a las creencias sobre los hechos como consecuencia de experiencias propias o ajenas, de la cultura, de prejuicios; pueden ser o no producto del conocimiento científico y posiblemente sean útiles para toma de decisiones de cada día. Las pseudociencias parten de observa- 
ciones asistemáticas y enuncian hipótesis explicativas que, a veces, son falacias, enunciados imposibles de comprobar o que, aunque puedan someterse a prueba, no se lo considera necesario. El conocimiento científico, en cambio, se caracteriza por ser "racional, sistemático, exacto, verificable y por consiguiente falible" (Bunge, 1963, p. 7). Además, al tener conciencia de sus limitaciones, es un sistema abierto y corregible. La diferencia fundamental entre pseudociencias, sentido común y ciencia consiste en que esta última va construyendo conceptos del universo cada vez más amplios, profundos y exactos porque sus hipótesis las somete a pruebas empíricas para controlar, aunque no eliminar, la subjetividad. De este modo, llega a conformar sistemas de conocimiento (teorías y leyes) cada vez más alejadas de la realidad tangible.

Se considera que el aprendizaje del pensamiento científico no es una tarea sencilla ya que no es un tipo de pensamiento natural en la especie humana (por ej., McCauley, 2011; Wolpert, 1992). Se deben cambiar ciertas estructuras del conocimiento hacia nociones que implican reflexión y una transformación de la red conceptual, ya que algunas son parte del procesamiento de información que está en la naturaleza de todos los organismos vivos. Por ejemplo, las percepciones directas nos engañan mostrando que la tierra es plana y que todo gira alrededor de ella (teoría geocéntrica). Se necesitó mucha acumulación de evidencias indirectas y sistemáticas para comprobar lo contrario. Además, todos los animales, para sobrevivir, tienen estructuras que le permiten asociar acontecimientos externos que, si aparecen uno a continuación del otro con cierta frecuencia, tienden a inferir que el primero es causa del segundo, cuestión que no siempre es válida cuando esas creencias se someten a pruebas controladas (e.g., Skinner, 1948). En este sentido, en psicología experimental existe el efecto de la ilusión del control. Consiste en que los sujetos tienden a creer que pueden controlar o ser causa de algunos acontecimientos en los que claramente no tienen ninguna influen- cia. Solamente los estudios de laboratorio pudieron dar cuenta de este fenómeno y le dieron diversas explicaciones teóricas relacionadas con el aprendizaje asociativo (por ej., Jenkins \& Ward, 1965; Matute, 2002). Actualmente se considera que este mecanismo puede ser lo que da origen a las pseudociencias (por ej., Vadillo, Matute \& Blanco 2013). La ciencia, en resumen, puede comenzar por observaciones directas y conjeturas del sentido común, e incluso de las pseudociencias, pero luego se aleja de ellas creando teorías y leyes abstractas. Por otra parte, el conocimiento científico permite predecir hechos y modificar el ambiente generando tecnologías y desarrollo de prácticas con mayor eficiencia, como la ingeniería o las recientemente llamadas Prácticas Basadas en la Evidencia (PBE; ver su aplicación a las psicoterapias en Mustaca, 2014). Si bien el conocimiento del sentido común es a veces necesario y el de las pseudociencias inocuo, pueden también derivar en creencias fanáticas y dogmáticas que llegan a provocan grandes desastres. Además, las personas pueden tomar decisiones erróneas que pueden provocarle problemas o hasta la muerte, por acudir, por ejemplo, a tratamientos médicos o psicológicos no validados.

El cambio conceptual de la psicología popular (o del sentido común) y de las pseudociencias hacia la ciencia psicológica también es difícil de enseñar, quizá mucho más que en la física o la biología, ya que estas últimas están históricamente más desarrolladas, más alejadas de las problemáticas humanas y existen menos controversias epistemológicas sobre su metodología de estudio. La psicología popular está constituida por las ideas o creencias que las personas utilizan durante la vida diaria para explicar su conducta y la de los otros. Se basan en el pensamiento intuitivo, en las experiencias personales o de los otros, en sus creencias, malas interpretaciones de teorías psicológicas que pertenecen al acervo cultural y que, en la mayoría de los casos, no se han comprobado. Algunas creencias populares y erróneas son: "ser positivo cura el 
cáncer", "quienes sufren de abuso sexual en la infancia, en la adultez siempre desarrollan trastornos de personalidad", etcétera. (ver Lilienfeld, Lynn, Ruscio, \& Beyestein, 2012). En resumen, los objetivos fundamentales en la enseñanza de la Psicología son: 1) lograr que los estudiantes aprendan a diferenciar lo que son datos de la ciencia psicológica de los que son creencias de la psicología popular o de las pseudociencias y 2) conocer y debatir los pseudoproblemas epistemológicos que aún perduran en el acervo cultural.

Una de las formas de aproximarse a averiguar si la enseñanza de la Psicología favorece el concepto de que es una disciplina científica, es evaluar la opinión de estudiantes y graduados acerca de su estatus científico. Existen estudios que indican que aún predominan en los estudiantes ideas erróneas sobre el tema. Gardner y Dalsing (1986) encontraron que los alumnos llegan a la universidad con conceptos falsos sobre el campo de la Psicología y que esas ideas permanecen, incluso después de tomar varios cursos durante la carrera. Estos autores pensaron que los estudiantes estaban propensos a adquirir sus creencias a través del medio ambiente y que, al ser parte del acervo cultural, son resistentes al cambio, aun cuando obtengan nociones opuestas basadas en la investigación sistemática. Friedrich (1996) observó que en los Estados Unidos la mayoría de las clases de Psicología hacían énfasis en los resultados de la investigación empírica, pero rara vez evaluaban el grado en que los estudiantes desarrollaban una noción clara de la importancia del uso del método científico para obtener esos conocimientos. Para evaluar las creencias sobre el estatus de la Psicología en alumnos y profesores, elaboró y validó la Escala de Percepción de la Psicología como ciencia (PAS; Friedrich, 1996), utilizada en la mayoría de las investigaciones posteriores sobre el tema y es la que se empleó en el estudio empírico de este artículo (ver el apartado Instrumentos). Morales, Abramson, Nai, Juniors y Bartoszeck (2005), utilizando la PAS, hallaron que los estudiantes de un curso de Psicología en una universidad del Norte de Brasil tenían ideas muy variadas sobre la disciplina. Si bien creían que la psicología es una ciencia, muy pocos estaban de acuerdo en que la investigación sirve para solucionar los problemas de la gente. Posteriormente, Bartoszeck, Abramson \& Place (2005) evaluaron, con el mismo instrumento, a 73 estudiantes de Psicología de la Universidad Nacional de Paraná, en el sur de Brasil. Encontraron que sólo el $47.8 \%$ estaba muy de acuerdo o de acuerdo con la idea que la psicología es una ciencia natural semejante a la biología o la física; el $33 \%$, que la investigación y la metodología científica es necesaria para llegar a la comprensión de la vida cotidiana y el $45 \%$, que la conducta es predecible. También se halló que la mayoría de los estudiantes desean ser psicólogos en áreas aplicadas; Malin y Timmreck (1979) informaron que el trabajo clínico y de asesoramiento es su meta más popular y que no hay indicios de que este patrón haya cambiado en las últimas tres décadas. Los estudiantes consideran que es prioritario aprender a ayudar a las personas por encima de los principios científicos y de la evaluación de las investigaciones aplicadas (McGovern \& Hawks, 1986), lo cual hace que las tendencias de los psicólogos aplicados sea no buscar sustentar sus prácticas en función de las investigaciones científicas.

En relación a los cambios de la percepción del estatus de la Psicología en los estudiantes en función de nivel de estudios o cantidad y tipo de materias cursadas, se encuentran distintos resultados. Holmes y Beins (2009) mostraron que, a través de los años de estudio, los estudiantes aumentaron sus conocimientos sobre la psicología, pero no incrementaron su percepción de la disciplina como una ciencia; frecuentemente creen que la observación sistemática es innecesaria para la comprensión humana, que es inferior a la experiencia personal y anecdótica y que el estudio de los resúmenes de los hallazgos de investigación es útil y que no se necesita examinar su metodología para evaluar su grado de validez. Además, 
al igual que Bartoszek y colaboradores (2005), hallaron que tenían ideas equivocadas sobre el estatus de la disciplina y se mantuvo después de varios años de estudios. En el mismo sentido, más recientemente, Pettijohn II, Pettijohn, Brenneman, \& colaboradores (2015), evaluaron con el PAS a estudiantes universitarios antes y después de realizar uno de cuatro cursos de psicología. Hallaron que los puntajes obtenidos antes eran semejantes en todos los alumnos; al final de los cursos, los puntajes del PAS aumentaron sólo en los que realizaron dos de ellos (Senior Tesis y Research methods), en otro disminuyó, aunque no significativamente (Introductory Psycho$\log y)$, y en el cuarto, decreció significativamente (Communication Psychology). Opuestos a esos hallazgos, Amsel, Baird y Ashley (2011) encontraron una correlación positiva entre la percepción de la psicología como ciencia y los años de estudio de alumnos de una universidad de EEUU. Igualmente, Bartels, Hinds, Glass y Rían (2009) hallaron que los estudiantes que habían tomado cuatro o más cursos de psicología tenían una percepción más favorable de la psicología como una ciencia en comparación con los que habían tomado hasta tres.

Respecto de la relación entre estudiantes y profesores, Holmes (2014) mostró que los profesores tenían más interés en la psicología como ciencia que los estudiantes y que estos últimos preferían las actividades prácticas de psicología aplicada (Estudio 1). Además, los que preferían esas actividades y estaban de acuerdo con que la intuición alcanzaba para la comprensión de la conducta humana, abandonaban los estudios universitarios con mayor probabilidad al cabo de los 2 años de la carrera (Estudio 2) que aquellos que opinaban lo contrario.

En Argentina no se realizaron investigaciones sobre las creencias de estudiantes y graduados sobre el estatus de la Psicología. Es de señalar que los planes de estudio de las carreras de psicología en ese país están organizados de modo que en los primeros años predomina la enseñanza de los procesos básicos, la estadística y la metodología de la investigación, dictados en general por docentes con alguna formación en investigación. En los últimos años de la carrera se desarrollan contenidos en áreas aplicadas, en especial de la clínica. Los docentes de estas materias en la mayoría de los casos están alejados de la investigación, tanto básica como aplicada (por ej, Ardila, 1978; Benito, 2009; García, 2009, Klappenbach, 2003; Mustaca, 2006). Además, la orientación predominante de casi todas las universidades es el psicoanálisis con todas sus variaciones.

El objetivo de la siguiente investigación es determinar y comparar qué opinión tienen sobre el estatus científico de la psicología 1) los estudiantes en distintos estadios de su carrera, y en función del género y edad, 2) los graduados de distintas orientaciones y actividad laboral, y 3 ) comparaciones entre los grupos. Se trata de un estudio exploratorio-descriptivo, cuantitativo y transversal que permitirá tener un cuadro de situación sobre el tema y ayudar a hacer cambios, si fuera necesario, en la enseñanza de la psicología.

\section{Método}

\section{Participantes}

Se tomó una muestra no probabilística de 492 estudiantes y graduados de psicología, de los cuales $197(40 \%)$ pertenecían a la Universidad Abierta Interamericana (UAI) y $231(43.3 \%)$ a la Universidad de Buenos Aires (UBA). El resto perteneció a otras universidades de Argentina e Iberoamérica: Chile $(n=7 ; 3 \%)$; España $(n=2 ; 1 \%)$ y Colombia, Ecuador, Méjico, Nicaragua y Perú $(n=1$ cada uno; $5 \%)$. Del total de los sujetos, $351(71.3 \%)$ eran mujeres y 141 $(28.7 \%)$, hombres. La muestra se dividió en dos grupos, uno de estudiantes $(n=419$; $85.32 \%)$ y otro de graduados $(n=73 ; 14.8 \%)$.

La edad promedio de los estudiantes fue de 27.18 años $(D E=7.59)$, de los cuales $306(73 \%)$ eran mujeres y $113(27 \%)$, va- 
rones. En cuanto a las instituciones a las que pertenecían, $184(43.9 \%)$ estudiaban en la UBA, $192(45.8 \%)$ en la UAI y $43(10.3 \%)$ en otras universidades. El promedio de materias aprobadas fue de 28.6. La mayoría estaba cursando cuarto y quinto año de la carrera $(n=225 ; 54.9 \%$ y $n=41 ; 10 \%$, respectivamente); el resto se concentró en primer año $(n=128 ; 31.2 \%)$ y la menor parte, entre segundo y tercer año $(n=5 ; 1.2 \%$ y $n=$ $7 ; 1.7 \%$, respectivamente). Finalmente, 324 estudiantes $(77.3 \%)$ preferían dedicarse a áreas aplicadas (clínica: 58.5\%, jurídica: 9.5\%, laboral: $4.3 \%$ y educacional: $5 \%), 14$ $(3.3 \%)$ a la investigación-psicología experimental y $81(19.3 \%)$ aún no lo sabían.

El grupo de graduados se conformó con 73 sujetos ( $36.7 \%$ del total de la muestra) de los cuales $45(61.6 \%)$ eran mujeres y 28 $(38.4 \%)$, varones; la edad promedio fue de 35.59 años $(D E=11.35)$; 58 graduados $(79.55 \%)$ estudiaron en universidades de Argentina, la mayoría en la UBA $(n=32$; $43.84 \%$ ) siguiendo la UAI, Universidad Nacional de Córdoba y Universidad Nacional de San Luís ( $n=5$ en cada una; $20.4 \%$ ); el resto se distribuyó en otras universidades de Argentina, Latinoamérica y España. Respecto del nivel de estudios, el 53.3\% $(n=$ 40) eran licenciados, el $22.7 \%(n=17)$ hizo alguna especialización, el 3.5\% $(n=7)$, una maestría y el $5.5 \%(n=11)$, un doctorado. En cuanto a las áreas de trabajo, el $42.7 \%$ $(n=32)$ se especializó en investigación y/o docencia y el $57.7 \%$, en áreas aplicadas (clínica y/o educacional: $28 \%, n=31$, en otras áreas: $29.3 \%, n=22$ ). Sobre la orientación, el $54.4 \%(n=31)$ manifestó tener una orientación cognitiva-comportamental, un 33.3\% $(n=19)$, integrativa-ecléctica y un $12.3 \%$ $(n=7)$, psicoanalítica.

\section{Instrumentos}

Se utilizaron dos cuestionarios:

1.- Escala de Psicología como Ciencia (PAS), basada en la construida por Friedrich (1996): Evalúa las creencias sobre los supuestos de la psicología. Contiene 20 ítems con respuestas tipo Likert con 7 pun- tos: desde 1 (Muy en desacuerdo) hasta 7 (Muy de acuerdo). La traducción al castellano fue realizada por los autores del trabajo, evaluada por dos expertos en psicología y con alto conocimiento del idioma inglés. Luego fue administrada a un grupo de estudiantes de la carrera de Psicología para confirmar la comprensión de los ítems. La escala está dividida en tres factores: F1, la psicología es una ciencia semejante a la biología o la física (4 ítems; por ej., Una licenciatura en Psicología debe ser una licenciatura en Ciencias en lugar de una en Artes o Humanidades); F2, la investigación llevada a cabo en entornos controlados de laboratorio es esencial para la comprensión de la conducta cotidiana ( 5 ítems, por ej., Es tan importante para los estudiantes de psicología que puedan hacer experimentos como lo es para los estudiantes de química, física y biología) y F3, la conducta es predecible (6 ítems, por ej., A pesar que cada persona es única, es posible para la ciencia encontrar leyes generales para explicar el comportamiento humano). Además, tiene 4 preguntas distractoras (Fr) para ensombrecer los objetivos de la encuesta y controlar la deseabilidad social (por ej.: Un curso de introducción a la psicología debe abarcar la más amplia gama de temas como sea posible). Algunos ítems se evalúan de manera invertida. Se considera que a mayor puntaje, mayor acuerdo que la psicología debe ser entendida como una ciencia fáctica. Friedrich (1996) informó que la escala tiene un coeficiente Alpha de consistencia interna de .71 para una muestra de estudiantes, y Holmes (2014) halló un Alpha de .74 para graduados-docentes. Para la muestra que se presenta en esta investigación, el Alpha de Cronbach fue de .74.

2. Cuestionario sociodemográfico. Se indagó edad, sexo y el desempeño actual de los estudiantes (en qué universidad cursa, en qué año de la carrera está, cantidad de materias aprobadas, orientación que piensa seguir), y de los graduados (en qué universidad estudió, qué nivel de grado alcanzó, años de trabajo en la disciplina, orientación y especialidad). 


\section{Procedimiento}

La encuesta se administró de dos maneras. Un grupo $(n=161 ; 32.7 \%)$ respondió de manera online y otro $(n=331 ; 67.3 \%)$ lo hizo de forma presencial. Previo al consentimiento informado, que garantizaba el anonimato de las respuestas y unos ejemplos de cómo se debía llenar el cuestionario, se presentaron las 20 preguntas y a posteriori el cuestionario sociodemográfico. Las encuestas online se enviaron por medio de Facebook y correo electrónico a estudiantes y graduados, como así también a instituciones de psicología de diversas especialidades y orientaciones, tales como la Asociación Psicoanalítica Argentina (APA), la Asociación Argentina de Ciencias del Comportamiento (AACC), la Asociación para el Avance de la Ciencia Psicológica (AACP), clínicas psicoanalíticas, sistémicas, eclécticas, etcétera. Las encuestas presenciales las llevó a cabo un estudiante de años avanzados para controlar posibles efectos de deseabilidad social si las administraba un docente.

Para el análisis de los resultados se utilizó el paquete SPSS v20.

\section{Resultados}

Los análisis inferenciales se realizaron en función de los ítems y luego de los factores. Se llevó a cabo el estadístico Kolmogorov-Smirnov para una muestra en las variables criterio. Se halló que los F3 y Fr poseían una distribución normal ( $p s>.05)$; en cambio, los F1 y F2 no la tenían ( $p s<.05)$. Debido a esto se utilizaron pruebas no paramétricas ( $U$ de Mann Whitney, KruskalWallis y la Correlación Rho de Spearman).

\section{Puntajes de cada ítem en función de estudiantes y graduados.}

La Figura 1 muestra el puntaje medio obtenido por los grupos en cada una de las 15 preguntas correspondientes a los factores 1,2 y 3 . Se observa que los graduados se establecieron por encima de los estudiantes. El promedio general de los estudiantes fue de $4.68(D E=.92)$ y el de los graduados de $5.69(D E=.68)$. Un análisis de los promedios generales utilizando la $U$ de Mann Whitney arroja una diferencia significativa entre estudiantes y graduados $(U=212 ; p<$ .002). Los puntajes menores a 4 de los estudiantes corresponden a los ítems 8, 9, 10, 17,18 y 20 . Esto se puede interpretar como que los estudiantes opinan que la realización de investigaciones controladas de laboratorio no es tan útil para solucionar problemas psicológicos, que la libertad para comportarnos no permite predecir la conducta, que los consejos psicológicos en revistas populares son útiles como los basados en las investigaciones psicológicas, que los psicólogos en áreas aplicadas no necesitan interesarse en los resultados de las investigaciones, que las teorías psicológicas que se presentan en los medios de comunicación se pueden tomar como confiables, y que los estudiantes no obtienen beneficios con aprender los procedimientos para la realización de investigaciones. Sin embargo, estaban muy de acuerdo con que "el estudio de la psicología debe considerarse principalmente como una ciencia" (ítem 13; promedio: 6.04). Entre los graduados, los puntajes medios más bajos fueron 5.23 (ítem 16: La investigación psicológica puede llegar a permitirnos anticipar el comportamiento de las personas con un alto grado de precisión) y 5.37 (ítem 6: Las investigaciones controladas realizadas en un laboratorio son esenciales para comprender y poder explicar el comportamiento cotidiano y patológico). Los promedios más altos fueron 6.49 (ítem 12: La financiación de los gobiernos para la investigación psicológica controlada es tan necesaria para el desarrollo de lo que sabemos sobre el comportamiento como lo es para la adquisición de conocimientos en áreas como la química, la física o la biología) y 6.47 (ítem 17: Los psicólogos que trabajan como profesionales en áreas aplicadas tienen que estar interesados en los resultados de las investigaciones en psicología y neurociencias). 
Comparación de los puntajes de los factores en estudiantes de la UBA vs UAI y entre género.

Los puntajes obtenidos entre los estudiantes de la UBA y la UAI no presentan diferencias significativas en ninguno de los factores $(p>.05)$, por lo cual los análisis posteriores se realizaron con la muestra total de los alumnos. Por otra parte, un análisis comparativo entre los puntajes y género indica una diferencia significativa a favor de los varones $(\mathrm{F} 1: Z=-4.03, p<.001 ; \mathrm{F} 2$ : $Z=-3.07, p<.01 ; \mathrm{F} 3: Z=-4.92, p<.001)$.

\section{Porcentaje de estudiantes y gradua- dos con puntajes de 6 y 7 .}

Se calculó el porcentaje de estudiantes y de graduados que puntuó 6 o 7 (de acuerdo o completamente de acuerdo, según Bartoszeck \& colaboradores, 2005) en todos ítems de cada uno de los tres factores de interés del PAS (ver Figura 2). Por ejemplo, en el F1 tenían que marcar en sus cuatro ítems las opciones de respuesta correspondientes a 6 ó 7 para ser contabilizados. A partir de este análisis, se observa que en ambos grupos el porcentaje de 6 ó 7 en las preguntas de cada factor es bajo, aunque en los estudiantes lo es aún más. De los estudiantes, el $25 \%(n=105)$ puntúa 6 ó 7 en F1, el $10.7 \%(n=45)$ en F2 y sólo el $5 \%(n=$ $21)$ en F3. De los graduados, el $53.4 \%(n=$ 39) puntúa 6 ó 7 en F1, el 45.2\% $(n=33)$ en F2 y el $20.5 \%(n=15)$ en F3.

Relación entre los puntajes de los factores y la edad, género y años de estudio en los estudiantes.

En el grupo de estudiantes se halló que la edad correlaciona significativamente negativa con F2 $(r=-.16 ; p<.01), \mathrm{F} 3(r=-.13$; $p<.01)$ y $\mathrm{FR}(r=-.12 ; p<.05)$. En cuanto al género, al igual que en la muestra total, los hombres obtienen un mayor puntaje que las mujeres en el F1 $(Z=-4.72 ; p<.001)$, en el F2 $(Z=-2.75 ; p<.01)$ y en el F3 $(Z=-4.42$; $p<.001)$.
Para evaluar la relación entre los puntajes según los años de estudio, se dividió a los estudiantes en dos grupos: 1) los primeros años de la carrera (estudiantes de primero, segundo y tercer año) y 2) los de cuarto y quinto año (últimos años de la carrera). La Figura 3 muestra el promedio de los puntajes en función de los dos grupos. Aunque se observa una diferencia pequeña entre los grupos, los análisis estadísticos arrojaron una diferencia significativa a favor de los estudiantes de los primeros años en el F1 $(Z=-2.35 ; p<.05), \mathrm{F} 3(Z=-5.43$; $p<.001)$ y $\operatorname{FR}(Z=-10.06, p<.001)$.

Relaciones entre los puntajes de los factores y las especialidades de los graduados.

Las especialidades de los graduados se clasificaron en tres grupos: 1) docentes-investigadores, 2) clínica-educacional y, 3) ambas categorías. El primer grupo obtuvo el mayor puntaje en los tres factores (F1= $6.32 ; \mathrm{F} 2=6.39$ y $\mathrm{F} 3=5.96)$; le sigue el grupo $3(\mathrm{~F} 1=6.01 ; \mathrm{F} 2=6.12$ y $\mathrm{F} 3=5.57)$ y el grupo $2(\mathrm{~F} 1=5.61, \mathrm{~F} 2=6.02$ y $\mathrm{F} 3=5.51)$. Estas diferencias no fueron significativas.

Relaciones entre los puntajes de los factores en estudiantes y la orientación de los graduados.

Finalmente, los puntajes se compararon entre los estudiantes y la orientación de los graduados: cognitivo-conductual, integrativa-ecléctica y psicoanalítica. La Figura 4 muestra el puntaje medio en función de los factores y los grupos. Los graduados que se identificaron con la orientación cognitivacomportamental mostraron un promedio que se sitúa entre las categorías muy de acuerdo o completamente de acuerdo en los tres factores $(\mathrm{F} 1=6.47 ; \mathrm{F} 2=6.45 ; \mathrm{F} 3=6.01)$; los integrativos-eclécticos, alrededor de 5 $(\mathrm{F} 1=5.83 ; \mathrm{F} 2=5.85 ; \mathrm{F} 3=5.57)$, y los psicoanalistas, entre 4 y $5(\mathrm{~F} 1=4.21 ; \mathrm{F} 2=5.63$; $\mathrm{F} 3=4.38$ ). Los estudiantes obtuvieron puntajes superiores a los psicoanalistas en F1 y $\mathrm{F} 3$ e inferiores a los otros dos grupos en los 
tres factores $(\mathrm{F} 1=5.68 ; \mathrm{F} 2=5.44 ; \mathrm{F} 3=5.12)$. La prueba $H$ de Kruskal-Wallis arrojó una diferencia significativa en el F1 $\left(\chi^{2}(2)=\right.$ 42.61; $p<.001), \mathrm{F} 2\left(\chi^{2}(2)=65.13 ; p<.001\right)$ y en el F3 $\left(\chi^{2}=43.05 ; p<.01\right)$. Luego se comparó cada grupo con otro usando la prueba $U$ de Mann-Whitney. No se hallaron diferencias significativas entre los cognitivos-conductuales vs los integrativos - eclécticos en ningún factor ( $p s>.05)$; entre los cognitivos-conductuales vs los estudiantes se hallaron diferencias significativas a favor del primero en los tres factores $(p<.01)$. Lo mismo ocurrió entre los cognitivos-comportamentales vs los psicoanalistas $(p<.01)$; entre los integrativos-eclécticos vs los psicoanalistas se halló una diferencia en los factores 1 y $3(p<.05)$. Finalmente, entre los psicoanalistas vs los estudiantes se halló una diferencia significativa en el F1 a favor de los últimos $(p<.01)$.

\section{Discusión}

En la enseñanza de psicología uno de los objetivos primordiales es que los alumnos aprendan a discriminar entre los datos provenientes del conocimiento científico de aquellos derivados de las pseudociencias o de la psicología popular. Por lo cual una de las primeras cuestiones es que, tanto los estudiantes como graduados, deberían estar de acuerdo en que la psicología debe tener las características de una ciencia fáctica. Este trabajo tuvo como propósito conocer las opiniones de los estudiantes y graduados sobre el estatus científico de la psicología en Argentina, un tema que adquiere cada vez más relevancia dado el aumento constante del conocimiento de la conducta y de sus correlatos fisioneurobiológicos, evolutivos y sociales, tanto a nivel teórico como aplicado.

Con respecto a la muestra, se observa un predominio de graduados dedicados a la psicología aplicada, aunque responden docentes-investigadores que parecen solaparse con la orientación cognitiva conductual y la integrativa en el área aplicada (las categorías especialización y orientación no eran excluyentes). La mayor parte de los graduados fueron de orientación cognitiva-conductual, siendo escasa la participación de los psicoanalistas (7 sujetos), por lo cual hay que tomar con extrema precaución los resultados de esta orientación obtenidos en la muestra. Esta poca participación es paradójica dado que en Argentina predomina este último enfoque y que, teniendo en cuenta este hecho, en la distribución de las encuestas se tuvo un especial cuidado de enviarlas a psicoanalistas reconocidos y a numerosas instituciones, blogs y Facebook que cobijan a estos profesionales. Por otra parte, tanto los graduados como los estudiantes fueron en su mayoría mujeres y los estudiantes prefieren en el futuro dedicarse a áreas aplicadas. Estos últimos resultados son análogos a los datos obtenidos en otros países (ver Introducción).

Los puntajes medios obtenidos en estudiantes y graduados son relativamente parecidos a los informados por otros autores (e.g., Bartoszeck et al., 2005; Holmes, 2014; Pettijohn et al., 2015). Además, a semejanza del estudio de Holmes (2014), los estudiantes muestran puntajes significativamente menores que los graduados en los tres factores de interés del PAS (Figuras 1 y 2). En el ítem 13 (El estudio de la psicología debe considerarse principalmente como una ciencia) los estudiantes tienen un puntaje alto; sin embargo, en algunos de los ítems que detalla lo que trae aparejada la afirmación anterior, obtienen puntajes menores a 4. Esta incoherencia sugiere que los alumnos no tienen claridad sobre el concepto de ciencia.

El porcentaje de participantes que puntúa con 6 ó 7 en todos los ítems de cada factor de interés (de acuerdo o muy de acuerdo) es bajo, tanto en graduados como en estudiantes, aunque más en estos últimos. Este análisis e interpretación del significado de 6 y 7 aparece sólo en el artículo de Bartoszeck y colaboradores (2005) realizado con estudiantes en una universidad de Brasil. Los porcentajes de los estudiantes de esta mues- 
tra son más bajos que los que evaluaron en Brasil y no se pueden hacer comparaciones con otros países en relación a los graduados. El porcentaje de estos últimos es mayor que los de los estudiantes. Alrededor del 50\% de los graduados está de acuerdo o muy de acuerdo con que la psicología es una ciencia análoga a la biología o la física, y que la investigación es fundamental para su estudio, pero solamente el $20.5 \%$ considera que la conducta es predecible. Uniendo estos resultados con los sintetizados en la Figura 4 , donde se muestran los puntajes en función de la orientación de los graduados, se encuentra que los que se identifican como cognitivos-conductuales, obtienen puntajes medios de alrededor de 6 en los tres factores, por lo cual se puede inferir que estos últimos tienen una mayor coherencia en la concepción de la psicología como una ciencia fáctica, a diferencia de las otras orientaciones.

Entre los estudiantes uno de los resultados más sorprendentes es que en los últimos años de la carrera, los puntajes del F1 y F3 disminuyeron significativamente respecto de los primeros años, aunque las diferencias fueron pequeñas. Estos datos son opuestos a los que hallaron Amsel y colaboradores (2011) y Bartels y colaboradores (2009) y semejantes a los de Pettijohn y colaboradores (2015) en uno de los cursos que realizaron los alumnos y a Holmes y Beins (2009). Existen algunas investigaciones que hallaron que los cursos de estadística y metodología de la investigación se asocian con un aumento en el pensamiento crítico (por ej., Amsel et al., 2011; Friedrich \& Camac, 2003; Holmes \& Beins, 2009). Se podría conjeturar que el resultado hallado está determinado en cómo está configurado el plan de estudios de las universidades de Argentina y su orientación predominante en las áreas de aplicación (ver Introducción).
Este trabajo tiene alcances y algunas limitaciones que deben tenerse en cuenta. La muestra no es representativa, el número de los grupos de comparación no es homogéneo, y el cuestionario no fue validado en la versión en castellano. Por otra parte, esta investigación es la única que discriminó entre las opiniones de los profesionales de distintas orientaciones y la que evaluó cuál es el porcentaje de graduados que obtuvo el puntaje máximo en los factores. El resultado obtenido sugiere que coexisten al menos dos clases de opiniones sobre la psicología: una que la considera con todos los atributos de una ciencia fáctica y otra que sería una disciplina diferente a ella. En este sentido, Kimble (1984) realizó un estudio con otra prueba donde proporciona datos que muestra la existencia de dos culturas dentro de la psicología: la científica y la humanista, afirmando que la "psicología tiene un problema de identidad" (p. 833, traducción de los autores). Aparentemente esta dicotomía continúa vigente e influye en la enseñanza de la disciplina.

En resumen, este estudio permitió detectar situaciones que deben alertar a los psicólogos y especialmente a los docentes de la carrera de psicología en Argentina, e invita a debatir sobre su enseñanza y sobre todo a que se unifiquen las opiniones sobre el estatus de la psicología, ya que parecen perdurar serias controversias sobre el tema que pueden confundir y perjudicar a los futuros profesionales.

En el futuro convendría validar el instrumento y replicar el estudio con muestras más representativas. Además, se pueden iniciar estudios experimentales longitudinales que evalúen las opiniones de los estudiantes y docentes sobre el estatus de la psicología antes y después de participar en talleres de discusión sobre el tema. 


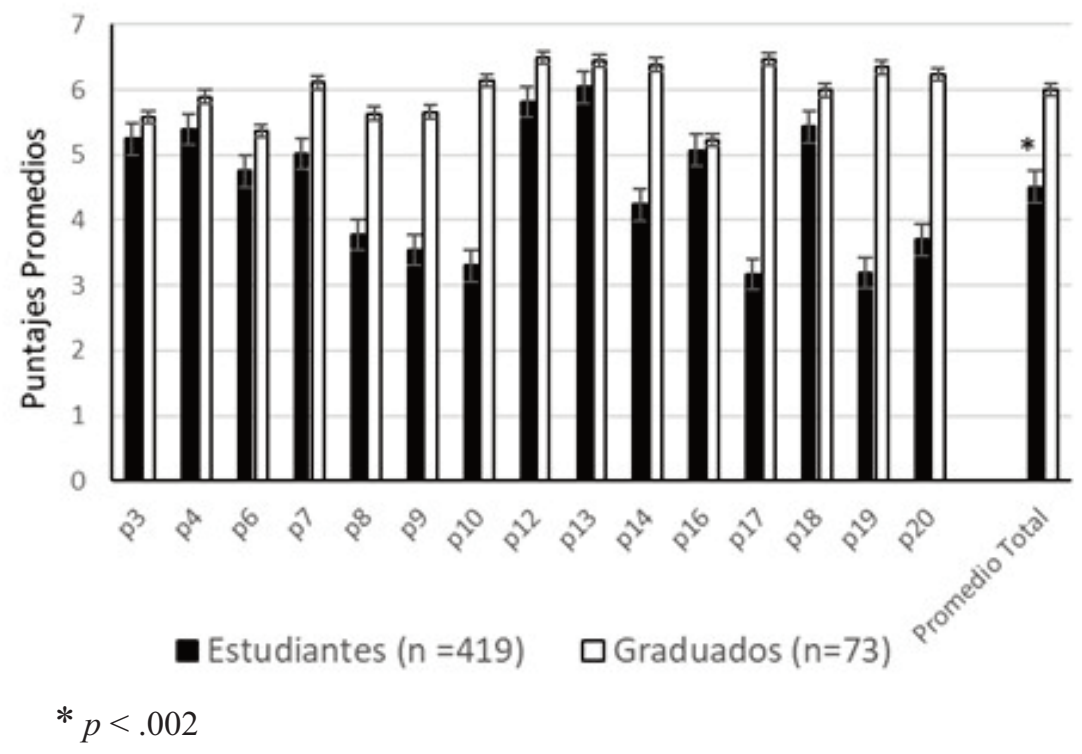

Figura 1. Promedio (SEM) del puntaje de los ítems la Escala PAS (Friedrich, 1996) según los grupos, descartando los correspondientes al factor relleno.

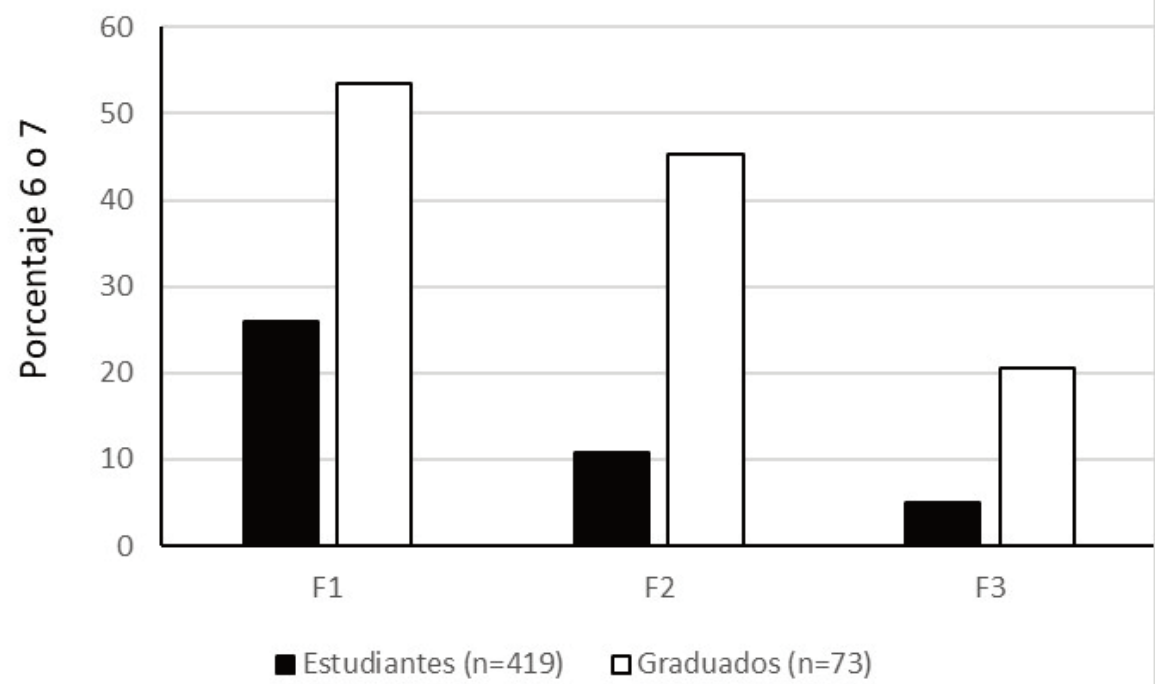

Figura 2. Porcentaje de los estudiantes y graduados que respondieron con un puntaje de 6 ó 7 (de acuerdo o completamente de acuerdo) a los ítems en función de los factores de Escala PAS (Friedrich, 1996). F1: La psicología puede considerarse como una ciencia semejante a la biología o la física; F2: La investigación llevada a cabo en entornos controlados de laboratorio es esencial para la comprensión de la conducta cotidiana; F3: La conducta es predecible. 


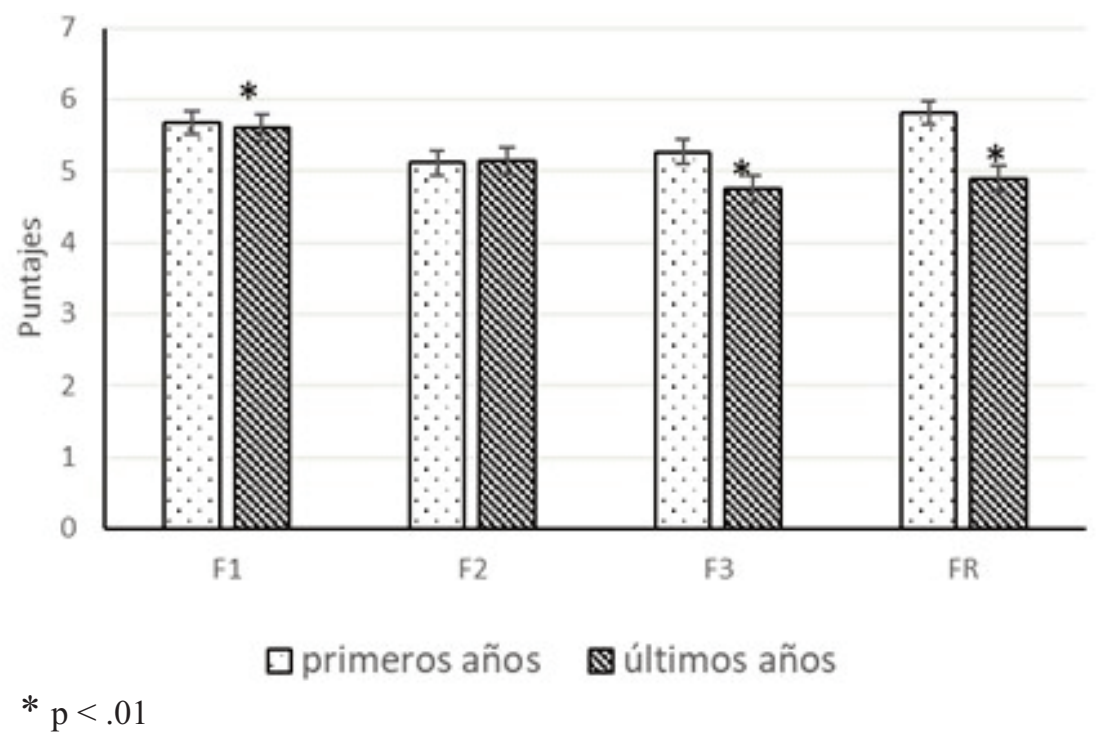

Figura 3. Promedio (SEM) de los puntajes de la Escala PAS (Friedrich, 1996) en función de los factores y de los años de estudio de los estudiantes. F1: La psicología puede considerarse como una ciencia semejante a la biología o la física; F2: La investigación llevada a cabo en entornos controlados de laboratorio es esencial para la comprensión de la conducta cotidiana; F3: La conducta es predecible, FR: de relleno.

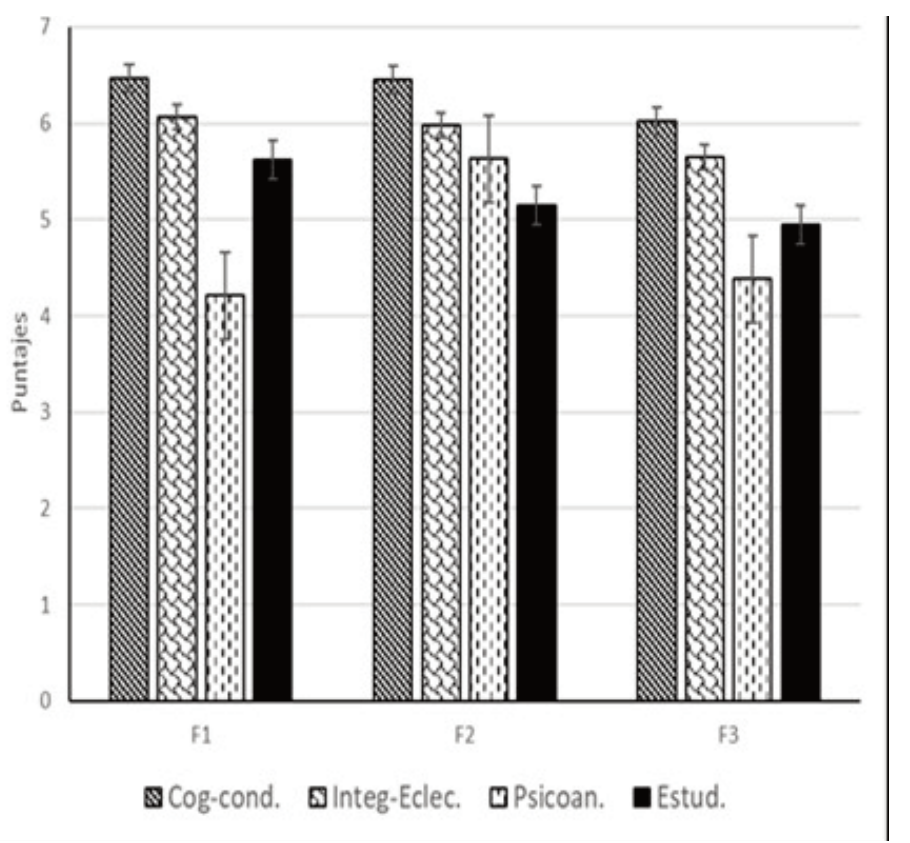

Figura 4. Promedio (SEM)) de los puntajes de la Escala PAS (Friedrich, 1996) en función de los factores de todos los estudiantes vs. la orientación profesional de los graduados: cognitivoconductual, integrativa-ecléctica y psicoanalítica. F1: La psicología puede considerarse como una ciencia semejante a la biología o la física; F2: La investigación llevada a cabo en entornos controlados de laboratorio es esencial para la comprensión de la conducta cotidiana; F3: La conducta es predecible. 


\section{Referencias bibliográficas}

Amsel, E., Baird, T. \& Ashley, A. (2011). Misconceptions and Conceptual Change in Undergraduate Students' Understanding of Psychology as a Science Psychology. Learning and Teaching, 10(1), 3-10. http:// dx.doi.org/10.2466/PR0.105.2.383-388

Ardila, R. (1978). La profesión del psicólogo. México: Trillas.

Bartels, J.M., Hinds, R.M., Glass, L.A., \& Ryan, J.J. (2009). Perceptions of psychology as a science among university students: The influence of psychology courses and major of study. Psychological Reports, 105(2), 383-388. http://dx.doi.org/10.2466/PR0.10 5.2.383-388

Bartoszeck, A., Abramson, C., \& Place, A. (2005). Perception of students in the south of Brazil of status of psychology as a science. Psychological Reports, 97(3), 750756. http://dx.doi.org/10.2466/pr0.97.3.750756

Benito, E. (2009). La formación en Psicología: revisión y perspectivas. Revista Psiencia, 1(2), 1-12. http://dx.doi.org/10.5872/ psiencia.v1i2.16

Bunge, M. (1963). La ciencia, su método y su filosofía. Buenos Aires: Ediciones Siglo 20.

Friedrich, J. (1996). Assessing students' perceptions of psychology as a science: Validation of a self-report measure. Teaching of Psychology, 23(1), 6-13. http://dx.doi.org/ 10.1207/s15328023 top2301_1

Friedrich, J., \& Camac, M. K. (2003). Psychologist as scientist or intuitive judge? Assessing student perceptions and associated reasoning strategies. Representative Research in Social Psychology, 27(1), 1-10.

García, L. N. (2009). La disciplina que no es: los déficits en la formación del psicólogo argentino. Revista Psiencia, 1(2), 12-17. http://dx.doi.org/10.5872/psiencia.v1i2.17

Gardner, R. M., \& Dalsing, S. (1986). Misconceptions about psychology among college students. Teaching of Psychology, 13(1), 3234. http://dx.doi.org/10.1207/s15328023top 13019

Holmes, J. D. (2014). Undergraduate Psychology's Scientific Identity Dilemma: Student and Instructor Interests and Attitudes. Teaching of Psychology, 41(2) 104-109. http://dx.doi.org/10.1177/009862831453 0339

Holmes, J. D., \& Beins, B. C. (2009). Psychology is a science: At least some students think so. Teaching of Psychology, 36(2), 511. http://dx.doi.org/10.1080/00986280802 529350

Jenkins, H. H. \& Ward, W. C. (1965). Judgement of contingency between responses and outcomes. Psychological Monographs, 79(1), 1-57. http://dx.doi.org/10.1037/h009 3874

Kimble, G.A. (1984). Psychology's Two Cultures. American Psychologist, 39(8), 833-939. http://dx.doi.org/10.1037/0003-06 6X.39.8.833

Klappenbach, H. (2003). La globalización y la enseñanza de la psicología en la Argentina. Psicología em Estudo, 8(2), 3-8.

Lilienfeld, S. O., Lynn, S.J., Ruscio, J., \& Beyestein, B.L. (2012). 50 grandes mitos de la psicología popular. Confusiones generalizadas e ideas erróneas sobre el comportamiento humano. Méjico: Océano.

Malin, J. T. \& Timmreck, C. (1979). Student goals and undergraduate curriculums. Teaching of Psychology, 3(1), 136-139. http://dx.doi.org/10.1207/s15328023top060 3_2

Matute, E. (2002). Introducción: Aprendizaje de relaciones causales. Cognitiva, 14(1), 714.

McCauley, R. (2011). Why religion is natural and science is not. Oxford, England: Oxford University Press.

McGovern, T. V., \& Hawks, B. K. (1986).The varieties of undergraduate experience. 
Teaching of Psychology, 13(2), 174-181. http://dx.doi.org/10.1207/s15328023top 130 4_1

Morales, B.L., Abramson, C.I., Nain, S., Nelson, A.S. Jr., \& Bartoszcck, A.B. (2005). Status of psychology as a science in Northeast Brazil: Undergraduate students' perceptions. Psychological Reports, 96(1), 109-114. http://dx.doi.org/10.2466/pr0.96.1. 109-114

Mustaca, A. (2006). La psicología científica y el análisis del comportamiento en Argentina. Avances de Psicología Latinoamericana, 24, 13-27. http://dx.doi.org/10.12804/ revistas.urosario.edu.co/apl/a.1227

Mustaca, A. (2014). Prácticas basadas en la evidencia: pasado, presente, futuro. Revista Costarricense de Psicología, 33(2), 93-108.

Pettijohn II, T., Pettijohn, T.F.; Brenneman, M., Glass, J.N., Brito, G., Terranova, A., Kim,
J., Meyersburg, C.A., \& Piroch, J. (2015). College student perceptions of psychology as a science as a function of psychology course enrollment. College Student Journal, 49(3), 461-465.

Skinner, B.F. (1948)."Superstition" in de pigeon. Journal of Experimental Psychology, 38(2), 168-172. http://dx.doi.org/10.1037/ 0096-3445.121.3.273

Vadillo, M.A., Matute, H., \& Blanco, F. (2013). Fighting the Illusion of Control: How to Make Use of Cue Competition and Alternative Explanations. Universitas Psychologica, 12(1), 260-269.

Wolpert, L. (1992). The unnatural nature of science. Cambridge, MA: Harvard University Press.

Recibido: 3 de noviembre de 2016 Aceptado: 15 de junio de 2018 